\title{
Inflammation-Induced Atherosclerosis as a Target for Prevention of Cardiovascular Diseases from Early Life
}

\author{
Roya Kelishadi* $^{*}$
}

\author{
Pediatric Preventive Cardiology Department, Isfahan Cardiovascular Research Centre, Isfahan University of Medical \\ Sciences, Isfahan, Iran
}

\begin{abstract}
Atherogenesis starts from the fetal life, and its natural course consists of interrelations between traditional risk factors and inflammatory, immune, and endothelial biomarkers. Even the early-stages of atherosclerotic lesions, i.e. fatty streaks present the features of chronic inflammation. Markers of inflammation are associated with insulin resistance and major atherosclerosis risk factors. Several studies have confirmed a relationship between surrogate markers of future cardiovascular disease with childhood obesity, notably abdominal obesity, as well as with the degree of obesity. Moreover, functional and structural changes are documented in arteries of children with a familial predisposition to atherosclerotic diseases; these changes are associated with clusters of inflammatory factors and markers of oxidation. In addition to the development of atheromatous plaques, inflammation also plays an essential role in the destabilization of artery plaques, and in turn in the occurrence of acute thrombo-embolic disorders. Markers of inflammation can provide predictive clinical information about outcomes of patients with acute coronary syndromes, independent of the extent of myocardial damage. Moreover, serum levels of the inflammatory markers might add prognostic information provided by traditional risk factors. Platelets have an important role in vascular inflammation and atherosclerosis and in the formation of mural thrombi. As lifestyle modification trials have been successful in decreasing endothelial dysfunction and the level of markers of inflammation among children and adolescents, it is suggested that in addition to expanding pharmacological therapies considered for secondary prevention of atherosclerotic diseases aiming to control the inflammatory process, the importance of primordial/primary prevention of atherosclerosis should be underscored.
\end{abstract}

Keywords: Atherosclerosis, Inflammation, Primordial, Primary and Secondary Prevention.

\section{INTRODUCTION}

Atherogenesis is the developmental process of atheromatous plaques characterized by a remodeling of arteries involving the concomitant accumulation of plaques. During the past decades, numerous theories or hypotheses have been proposed about atherogenesis that was previously considered a cholesterol storage disease. Although none of theories or hypotheses could entirely explain the whole process of the pathogenesis of atherosclerosis, but there is a growing body of evidence about the role of inflammation in this process. It is suggested that atherosclerosis is a specific form of chronic inflammatory process resulting from interactions between plasma lipoproteins, cellular components as monocyte/ macrophages, T lymphocytes, endothelial cells and smooth muscle cells as well as the extracellular matrix of the arteries [1-3].

The major risk factors of atherosclerosis induce the secretion of leukocyte soluble adhesion molecules, which facilitate the attachment of monocytes to endothelial cells, and chemotactic factors, which in turn promote the monocytes'

\footnotetext{
*Address correspondence to this author at the Pediatric Preventive Cardiology Department, Isfahan Cardiovascular Research Centre, Isfahan University of Medical Sciences, Isfahan, Iran; Tel: +98 311 3377881-8; Fax: +98 3113373435 ;

E-mails:kroya@aap.net, Kelishadi@med.mui.ac.ir
}

movement into the subintimal space. It is suggested that the transformation of monocytes into macrophages and the uptake of cholesterol lipoproteins initiate the production of fatty streaks. Additional damaging stimuli would maintain the accumulation of macrophages, mast cells, and activated $T$ cells in the growing atherosclerotic lesions $[4,5]$.

Inflammatory cytokines related to vascular inflammation stimulate the production of endothelial adhesion molecules, proteases, and other soluble mediators. They also induce the generation of the messenger cytokine interleukin-6, which in turn stimulates the liver to increase production of acutephase reactants such as C-reactive protein (CRP). In addition, platelets and adipose tissue can generate inflammatory mediators relevant to atherogenesis and thrombosis [6].

\section{INFLAMMATORY PROCESS OF ATHEROGENESIS FROM FETAL LIFE}

The first stages of atherogenesis begin from the fetal life. A local inflammatory process is suggested to contribute in reestablishment of the homeostasis of the vascular wall through promoting the removal of injured tissue and its consequent repair. The endothelial permeability of some arterial segments alters and allows the infiltration of macromolecules, like lipoproteins, in the subintimal space. Oxidized lipoproteins induce the expression of chemokines and adhesion molecules on the luminal surface of the 
endothelium, which allow the local recruitment of monocytes-macrophages and $\mathrm{T}$ lymphocytes [7].

Even the early-stages of atherosclerotic lesions, i.e. fatty streaks present the features of chronic inflammation. Atherogenic lipoproteins such as oxidized low density lipoprotein (ox- LDL), remnant lipoprotein and lipoprotein (a) have a significant role in the pro-inflammatory reaction, whereas high density lipoprotein (HDL), anti-atherogenic lipoproteins, have anti-inflammatory function [2, 5-7].

Changes in the proteoglycan metabolism of the intima are associated to the early stages of atherosclerosis. Matrix metalloproteinases (MMPs) are a group of zinc-dependent proteases produced by a variety of cell types, including endothelial, smooth muscle cells and monocytes. MMPs exist at low levels in normal adult tissue, and are synchronized in physiological and pathological remodeling processes. They are specialized enzymes involved in various stages of vascular diseases. The imbalance of matrix metalloproteinases (MMPs) and their inhibiting factors, i.e. tissue inhibitors of metalloproteinases (TIMPs) has a crucial role in early changes of atherosclerosis and its progression [8]. Experimental studies have shown that MMPs derived from arterial cells are required for early atherosclerotic plaque development and cellular accumulation $[9,10]$. MMPs are considered to mediate the progression of stable atherosclerotic lesions to an unstable plaque prone to rupture [11].

Angiotensin II is a proinflammatory mediator that induces the development and progression of atherosclerosis; it provokes pro-atherosclerotic cytokine and chemokine secretion and increases endothelial dysfunction. Angiotensin promotes macrophage migration into the vascular intima and elastin breaks, it is essential in beginning the atherosclerosis process [12]. Accordingly, the medications inhibiting the rennin-angiotensin system are being used both primary and secondary prevention of atherosclerotic cardiovascular disease [13].

\section{INFLAMMATORY BIOMARKERS AS PREDICTORS OF FUTURE ATHEROSCLEROSIS}

The association of inflammatory processes with the development of atherosclerosis provides important links between underlying mechanisms of atherogenesis and risk factors. Therefore, more interest has focused on studies about inflammatory biomarkers as predictors of future clinical events. Several studies have examined different circulating markers of inflammations, such as cytokines and adhesion molecules, as potential predictors of the present and the future risk of cardiovascular diseases, most studies have determined the following factors: ox-LDL, proinflammatory cytokines as interleukin-1 and tumor necrosis factor-, adhesion molecules as intercellular adhesion molecule-1 and selectins, inflammatory stimuli with hepatic effects as interleukin- 6 or the products of the hepatic stimulation, such as serum amyloid A (SAA), high-sensitivity CRP ( hs CRP) and a host of other acute-phase reactants and leukocyte count. Furthermore, soluble CD40 ligand, adiponectin, interleukin 18, and matrix metalloproteinase 9 are considered to provide additional information for cardiovascular risk stratification and prediction [6]. Elevated plasma levels of molecules such as soluble intercellular adhesion molecule-1, interleukin-6 and hs CRP have been found to represent inflammatory markers of future risk of atherosclerotic diseases. Among these factors, CRP has emerged as the most influential and available marker for clinical and epidemiological use.

An association between this sensitive marker of inflammation and the development of atherosclerotic disease has been documented in different experimental [14-17] and epidemiological studies [18-21]. Some epidemiological studies have confirmed that high-sensitivity CRP (hsCRP) might predict future atherosclerotic diseases among healthy individuals. Moreover, platelets and adipose tissue might produce inflammatory mediators related to atherothrombosis.

It is suggested that cholesterol itself may generate a proinflammatory process resulting in atherosclerosis [22]. When trapped in the intima, oxidized products of LDL-C might be active as pro-inflammatory mediators. Human and animal studies have suggested a strong correlation between the auto-antibodies against oxidized LDL and the extent of atherosclerosis [23]. Cholesterol synthesis is associated with interleukin-6 mediated inflammation, which in turn is the common causative factor for aging and age-related disorders including atherosclerosis $[24,25]$.

\section{INFLAMMATORY BIOMARKERS AND INSULIN RESISTANCE}

It is well documented that markers of the acute-phase response, including CRP are associated with insulin resistance [21-29], body mass index (BMI) [21, 26, 27, 29], serum glucose [26] and lipids [21, 26, 27, 29].

Serum hs CRP and interleukin-6 are independent risk factors for atherosclerosis in obese, insulin-resistant adults $[30,31]$. However, some studies have documented the association of CRP with cardiovascular disease, independent to insulin resistance [18-21]. Such studies have implicated inflammation as an essential contributor to the progression of atherosclerosis. Elevated serum levels of inflammatory markers, including CRP, are documented in type 2 diabetic patients with components of insulin resistance. It is suggested that insulin insensitivity and/or hyperinsulinemia may be associated with serum CRP levels [28].

CRP level is also related to the risk for future cardiovascular morbidity and mortality among those with documented vascular disease as well as apparently healthy individuals. It is noteworthy to mention that in some studies, the risk prediction of hs-CRP has been independent of traditional risk factors [18]. Longitudinal studies documented a predictive role of elevated circulating levels of soluble (s) intercellular cell adhesion molecule-1 (ICAM-1) in healthy people, of vascular cell adhesion molecule-1(sVCAM-1) in patients at high risk or with documented cardiovascular diseases [3234].

Generally, it is documented that all the conditions that increase circulating fatty acids and cause lipid overloading induce a lipotoxic state in non-adipose tissues that predispose insulin resistance and subsequent disorders [35]. 


\section{ASSOCIATION OF ENDOTHELIAL DYSFUNCTION AND INFLAMMATION FROM EARLY LIFE}

Atherogenesis starts from early life, and its natural course is an area under discussion of interrelations between traditional risk factors and inflammatory, immune, and endothelial biomarkers. Excessive production of CRP and other inflammatory biomarkers may accelerate atherogenesis in children and lead to long-term atherothrombotic events [36]. As endothelial dysfunction and atherosclerosis begin from early life, studying the association of their early stages with markers of inflammation would give a better insight of this process, independent of the process of aging. A study that determined the association of hs CRP with vascular endothelial and smooth muscle dysfunction in children with type 1 diabetes mellitus and healthy controls, hsCRP was not associated with early vascular dysfunction in normal-weight diabetic children, but such association was documented in those with higher BMI [37]. Another study investigated the relationship of hs CRP with BMI and cardiovascular risk factors in obese children and adolescents. It found that hs CRP level was correlated with BMI, systolic and diastolic blood pressure and serum leptin levels. It confirmed the relationship between obesity, inflammation and atherosclerosis, and suggested that hs CRP in childhood obesity might be a useful index to predict possible atherosclerotic events [38].

Studies on the relationship between the degree of childhood obesity and surrogate markers of future cardiovascular disease revealed that CRP and interleukin-6 levels increased with the degree of obesity. This finding suggests that the degree of low-grade inflammation may increase as children become more obese. However, C-reactive protein levels did not correlate significantly with insulin resistance or with the metabolic syndrome, suggesting that an underlying inflammation may be an additional factor contributing to adverse long-term cardiovascular outcomes [39].

Endothelial adhesion molecules, including endothelialleukocyte adhesion molecule (E-selectin), ICAM-1, and VCAM-1 have a crucial role in the earliest stages of atherogenesis by mediating the binding and recruitment of monocytes into arterial intima [40]. Concentrations of hs CRP and soluble adhesion molecules are higher in obese than in other children $[41,42]$. A population-based study among healthy children and adolescents found a significant correlation between CRP and markers of oxidative stress, as well as between CRP and these markers with abdominal obesity, but not with generalized obesity [43].

Childhood obesity and associated cardio-metabolic risk factors are no more limited to industrialized countries, but also a growing problem in low- and middle-income countries [44]. Therefore, future atherosclerotic diseases in young age would become a major health threat worldwide. Modifiable lifestyle behaviors are associated with childhood obesity [45] and cardio-metabolic risk factors [46]. Given that short-term lifestyle modification programs have been successful in improvement of lipid profile, markers of inflammation and endothelial dysfunction among obese youths [47, 48], early recognition and control of risk factors and in turn inflammation might have long-term impact on prevention and control of atherosclerosis and underlying inflammatory processes. Cardio-metabolic risk factors are not limited to obese indi- viduals, and are documented in some normal weight children and adolescents, termed phenotypically normal metabolically obese youths [49].

In addition to the lipids and apolipoproteins, the markers of inflammation and oxidative stress as well as the carotidintima media thickness of normal-weight children with a metabolic abnormality are found to be similar to obese children. Furthermore, irrespective of obesity and metabolic abnormality, fitness is shown to have the highest inverse correlation with markers of insulin resistance and nonalcoholic fatty liver diseases among adolescents [50].

Lifestyle modification trial has been successful in reducing serum CRP level among both normal-weight and obese children and adolescents with cardio-metabolic risk factors [51].

Given that cardio-metabolic risk factors track from early life to adulthood, and that childhood CRP value predict adult $\mathrm{CRP}$, and this association is found to be independent of other metabolic risk factors such as serum lipids, blood pressure, smoking, obesity indices, and insulin [52], and that high CRP is one of the youth determinants of adult metabolic syndrome [53], all children and adolescents, and not only those with overweight, should be encouraged to have healthier lifestyle that would help the primordial/primary prevention of atherosclerotic diseases.

\section{INFLAMMATORY CHANGES AND PRECURSORS OF ATHEROSCLEROSIS IN CHILDREN WITH POSITIVE FAMILY HISTORY OF PREMATURE CORONARY HEART DISEASE}

Offspring of patients with premature atherosclerotic disease may be at increased risk for atherosclerosis. Screening and control of atherosclerosis' risk factors are of special concern for these children who are at high-risk for premature atherosclerotic cardiovascular diseases. In addition to higher levels of lipids and lipoproteins [54], markers of oxidation [55], hs CRP [56] and immunologic factors [57], structural and functional changes in arteries are present at an early age in the arteries of individuals with a parental history of premature atherosclerosis $[56,58]$. It is documented that the 19year-old offspring of patients with premature myocardial infarction had lower flow-mediated reactivity of the brachial arteries and greater mean intima-media thickness of the common carotid artery. In those individuals with a parental history of premature myocardial infarction, an inverse association was found between brachial-artery reactivity and carotid intima-media thickness. This study also documented that both brachial-artery reactivity and carotid intima-media thickness were significantly and independently correlated with a parental history of premature myocardial infarction [58]. Vascular structural changes, as increased intima media thickness of the carotid artery, are detectable in children and adolescents, and might occur independently of traditional cardiovascular risk factors [59]. It is documented that in children and adolescents with parental history of premature coronary heart diseases, clusters of inflammatory factors and markers of oxidation as well as carotid-intima media thickness and left ventricular mass were correlated with each other and are associated with a positive parental history of premature coronary heart diseases in youths $[60,61]$. These 
findings complement the functional and structural changes documented in arteries of adults with a familial predisposition to atherosclerotic diseases.

CRP is one of the best markers for prediction of incident coronary artery diseases; this association is shown to be independent of other established risk factors [62]. CRP and oxidation are correlated; oxidative stress may be a determinant of CRP levels and promote the pro-atherosclerotic inflammatory process [63], and CRP can weakens the antioxidant defenses of endothelial progenitor cells and might predict future vascular events [64]. The relation of inflammatory factors and family history of coronary heart diseases among children and adolescents is a confirmatory finding that subclinical chronic inflammation may have a major role in the development of atherosclerosis. These findings underscore the importance of using a high-risk approach for primordial/primary prevention of atherosclerotic diseases from early life.

\section{INFLAMMATION AND THROMBOEMBOLISM}

Inflammation is not only instrumental in the development of atheromatous plaques, but also plays an essential role in the destabilization of artery plaques, and in turn altering chronic atherosclerosis into an acute thrombo-embolic disorder. A number of humoral factors including cytokines, cyclooxygenase-2, matrix metalloproteinases, and tissue factor are involved in the process of plaque destabilization [65].

A growing body of evidence indicate that the association of inflammation with atherosclerosis can provide predictive clinical information. Elevation in markers of inflammation predicts outcomes of patients with acute coronary syndromes, independent of the extent of myocardial damage. Moreover, serum levels of the inflammatory markers as CRP might add prognostic information provided by traditional risk factors [66, 67].

As proposed by Libby and Theroux, "the findings on the widespread nature of inflammation in patients prone to develop ACS challenge the traditional view of coronary atherosclerosis as a segmental or localized disease. Thus, treatment of acute coronary syndromes should involve two overlapping phases: first, addressing the culprit lesion, and second, aiming at rapid stabilization of other plaques that may produce recurrent events" [1]

Platelets have an important role in vascular inflammation and atherosclerosis. They exert essential role in modulating inflammatory and immune processes. During the early stages of atherosclerosis, platelets adhere to endothelial cells, and in later stages they participate in the formation of mural thrombi [68]. They can stimulate the secretion of chemokines in cells of the vascular wall cells [69]; some chemokines accelerate platelet aggregation and adhesion [70], in turn activated platelets release chemokines and precursors which trigger atherogenesis or modulate key processes such as angiogenesis and lipoprotein metabolism [71]. Platelet adhesion depends on the interaction of subendothelial matrix components, such as collagen, fibronectin and von Willebrand factor with other adhesive proteins platelet receptors. Platelet adhesion activates the platelet release of inflammatory substances that will result to firm platelet aggregation and thrombus formation [72]. As supposed by Weber, "An intricate functional relationship between platelets and chemokines emerges from the multiple interactions. The mechanisms provide a framework for synergistic functions and allow insights into the deleterious basis for proatherogenic, proinflammatory, or thrombogenic effects exerted by two prime suspects in the pathogenesis of vascular disease."[73]. Thus, pharmacological therapies considered for secondary prevention of atherosclerotic diseases aim to control the inflammatory process [74], and they need to be expanded.

\section{CONCLUSIONS}

Atherogenesis starts from the fetal life, and its natural course consists of interrelations between traditional risk factors and inflammatory, immune, and endothelial biomarkers. Some studies have suggested a role for genetic polymorphisms associated with inflammation and atherosclerosis $[75,76]$ but not confirmed by some others [77]. Because of considerable ethnic differences in the role of genetic in atherosclerosis [78], large multi-centric studies should be conducted in this regard.

As lifestyle modification trials have been successful in decreasing endothelial dysfunction and the level of markers of inflammation among children and adolescents, it is suggested that in addition to expanding pharmacological therapies considered for secondary prevention of atherosclerotic diseases aiming to control the inflammatory process, the importance of primordial/primary prevention of atherosclerosis $[79,80]$ should be underscored. Future studies are needed to find out more details about the role of inflammation in young ages, notably children and adolescents in order to improve strategies for prediction, prevention and treatment of atherosclerotic events.

\section{REFERENCES}

[1] Libby P, Theroux P. Pathophysiology of coronary artery disease. Circulation 2005; 111: 3481-8.

[2] Rodríguez G, Mago N, Rosa F. Role of inflammation in atherogenesis. Invest Clin 2009; 50: 109-29.

[3] Festa A, D'Agostino R Jr, Howard G, Mykkänen L, Tracy RP, Haffner SM. Chronic subclinical inflammation as part of the insulin resistance syndrome: the Insulin Resistance Atherosclerosis Study (IRAS). Circulation 2000; 102: 42-7.

[4] Tracy RP, Lemaitre RN, Psaty BM, et al. Relationship of Creactive protein to risk of cardiovascular disease in the elderly: results from the Cardiovascular Health Study and the Rural Health Promotion Project. Arterioscler Thromb Vasc Biol 1997; 17: 11217.

[5] Steffens S, Mach F. Inflammation and atherosclerosis. Herz 2004; 29: 741-8.

[6] Packard RR, Libby P. Inflammation in atherosclerosis: from vascular biology to biomarker discovery and risk prediction. Clin Chem 2008; 54: 24-38.

[7] Caligiuri G. Role of the immune response in atherosclerosis and acute coronary syndromes. Med Sci (Paris) 2004; 20:175-81.

[8] Kunz J. Matrix metalloproteinases and atherogenesis in dependence of age. Gerontology 2007; 53: 63-73.

[9] Yamada S, Wang KY, Tanimoto A, et al. Matrix metalloproteinase 12 accelerates the initiation of atherosclerosis and stimulates the progression of fatty streaks to fibrous plaques in transgenic rabbits. Am J Pathol 2008; 172: 1419-29.

[10] Choi ET, Collins ET, Marine LA, et al. Matrix metalloproteinase-9 modulation by resident arterial cells is responsible for injuryinduced accelerated atherosclerotic plaque development in apolipoprotein E-deficient mice. Arterioscler Thromb Vasc Biol 2005; 25: $1020-5$. 
[11] Johnson JL. Matrix metalloproteinases: influence on smooth muscle cells and atherosclerotic plaque stability. Expert Rev Cardiovasc Ther 2007; 5: 265-82.

[12] Nobuhiko A, Suganuma E, Babaev VR, et al. Angiotensin II amplifies macrophage-driven atherosclerosis. Arterioscler Thromb Vasc Biol 2004; 24: 2143-8.

[13] Montecucco F, Pende A, Mach F. The renin-angiotensin system modulates inflammatory processes in atherosclerosis: evidence from basic research and clinical studies. Mediators Inflamm 2009; 2009: 752406

[14] Pepys MB, Rowe IF, Baltz ML. C-reactive protein: binding to lipids and lipoproteins. Int Rev Exp Pathol 1982; 27: 83-111.

[15] Hatanaka K, Li XA, Masuda K, Yutani C, Yamamoto A. Immunohistochemical localization of $\mathrm{C}$-reactive protein binding sites in human aortic lesions by a modified streptavidin-biotin-staining method. Pathol Int 1995; 45: 635-41.

[16] Torzewski J, Torzewski M, Bowyer DE, et al. C- reactive protein frequently colocalizes with the terminal complement complex in the intima of early atherosclerotic lesions of human coronary arteries. Arterioscler Thromb Vasc Biol1998; 18: 1386-92.

[17] Cermak J, Key N, Bach R, Balla J, Jacob HS, Vercellotti GM. Creactive protein induces human peripheral blood monocytes to synthesize tissue factor. Blood 1993; 82: 513-20.

[18] Ridker PM, Cushman M, Stampfer MJ, Tracy RP, Hennekens CH. Inflammation, aspirin, and the risk of cardiovascular disease in apparently healthy men. N Engl J Med 1997; 336: 973-9.

[19] Haverkate F, Thompson SG, Pyke SD, Gallimore JR, Pepys MB, for the European Concerted Action on Thrombosis and Disabilities Angina Pectoris Study Group. Production of C-reactive protein and risk of coronary events in stable and unstable angina. Lancet 1997; 349: 462-6.

[20] Linton MF, Fazio S. Cyclooxygenase products and atherosclerosis. Drug Discov Today Ther Strateg 2008; 5: 25-36.

[21] Koenig W, Sund M, Fröhlich M, et al. C-reactive protein, a sensitive marker of inflammation, predicts future risk of coronary heart disease in initially healthy middle-aged men. Circulation1999; 99: 237-42.

[22] Paris D, Town T, Humphrey J, Yokota K, Mullan M. Cholesterol modulates vascular reactivity to endothelin-1 by stimulating a proinflammatory pathway. Biochem Biophys Res Commun 2000; 274 : 553-8.

[23] Hörkkö S, Binder CJ, Shaw PX, et al. Immunological responses to oxidized LDL. Free Radic Biol Med 2000; 28: 1771-9.

[24] Omoigui S. The Interleukin-6 inflammation pathway from cholesterol to aging--role of statins, bisphosphonates and plant polyphenols in aging and age-related diseases. Immun Ageing 2007; 4: 1

[25] Omoigui S. Cholesterol synthesis is the trigger and isoprenoid dependent interleukin-6 mediated inflammation is the common causative factor and therapeutic target for atherosclerotic vascular disease and age-related disorders including osteoporosis and type 2 diabetes. Med Hypotheses 2005; 65: 559-69.

[26] Mendall MA, Patel P, Ballam L, Strachan D, Northfield TC. C Reactive protein and its relation to cardiovascular risk factors: a population based cross sectional study. Br Med J 1996; 312: 1061-5.

[27] Tracy RP, Psaty BM, Macy E, et al. Lifetime smoking exposure affects the association of $\mathrm{C}$-reactive protein with cardiovascular disease risk factors and subclinical disease in healthy elderly subjects. Arterioscler Thromb Vasc Biol 1997; 17: 2167-76.

[28] Yudkin JS, Stehouwer CD, Emeis JJ, Coppack SW . C-reactive protein in healthy subjects: associations with obesity, insulin resistance, and endothelial dysfunction. Arterioscler Thromb Vasc Biol 1999; 19: 972-8.

[29] Festa A, D'Agostino R Jr, Mykkänen L, et al. Relative contribution of insulin and its precursors to fibrinogen and PAI-1 in a large population with different states of glucose tolerance: the Insulin Resistance Atherosclerosis Study (IRAS). Arterioscler Thromb Vasc Biol 1999; 19: 562-8.

[30] Blake GJ, Ridker PM. Inflammatory biomarkers and cardiovascular risk prediction. J Intern Med 2002; 252: 283-94.

[31] Tarantino G, Colicchio P, Conca P, et al. Young adult obese subjects with and without insulin resistance: what is the role of chronic inflammation and how to weigh it non-invasively? J Inflamm (Lond) 2009; 6: 6 .

[32] Peter K, Nawroth $\mathrm{P}$, Conradt C, et al. Circulating vascular cell adhesion molecule- 1 correlates with the extent of human atherosclerosis in contrast to circulating intercellular adhesion molecule-
1, E-selectin, P-selectin, and thrombomodulin. Arterioscler Thromb Vasc Biol 1997; 17: 505-12.

[33] Wilson AM, Ryan MC, Boyle AJ. The novel role of C-reactive protein in cardiovascular disease: risk marker or pathogen. Int $\mathrm{J}$ Cardiol 2006; 106: 291-7.

[34] Momiyama Y, Kawaguchi A, Kajiwara I, et al. Prognostic value of plasma high-sensitivity C-reactive protein levels in Japanese patients with stable coronary artery disease: The Japan NCVCCollaborative Inflammation Cohort (JNIC) Study. Atherosclerosis 2009 Apr 17. [Epub ahead of print]

[35] Lionetti L, Mollica MP, Lombardi A, Cavaliere G, Gifuni G, Barletta A. From chronic overnutrition to insulin resistance: the role of fat-storing capacity and inflammation. Nutr Metab Cardiovasc Dis 2009; 19: 146-52.

[36] Gasparyan AY, Mohammad-Hasani MR, Hassoun H, Darban H. Recent advances in cardiovascular medicine: the tenth Iranian congress on cardiovascular update. Arch Iran Med 2009; 12: 213-6.

[37] MacKenzie KE, Wiltshire EJ, Peña AS, et al. Hs-CRP is associated with weight, BMI, and female sex but not with endothelial function in children with type 1 diabetes. Pediatr Diabetes 2009 ;10: 44-51.

[38] Semiz S, Rota S, Ozdemir O, Ozdemir A, Kaptanoğlu B . Are Creactive protein and homocysteine cardiovascular risk factors in obese children and adolescents? Pediatr Int 2008; 50: 419-23.

[39] Ford ES. C-reactive protein concentration and cardiovascular disease risk factors in children: findings from the National Health and Nutrition Examination Survey 1999-2000. Circulation 2003; 108: 1053-8.

[40] Blankenberg S, Barbaux S, Tiret L. Adhesion molecules and atherosclerosis. Atherosclerosis 2003; 170: 191-203.

[41] Mangge H, Schauenstein K, Stroedter L, Griesl A, Maerz W, Borkenstein M. Low grade inflammation in juvenile obesity and type 1 diabetes associated with early signs of atherosclerosis. Exp Clin Endocrinol Diabetes 2004; 112: 378-82.

[42] Desideri G, De Simone M, Iughetti L, et al. Early activation of vascular endothelial cells and platelets in obese children. J Clin Endocrinol Metab 2005; 90: 3145-52.

[43] Kelishadi R, Sharifi M, Khosravi A, Adeli K. Relationship between $\mathrm{C}$-reactive protein and atherosclerotic risk factors and oxidative stress markers among young persons 10-18 years old. Clin Chem 2007; 53: 456-64.

[44] Kelishadi R. Childhood overweight, obesity, and the metabolic syndrome in developing countries. Epidemiol Rev 2007; 29: 6276.

[45] Kelishadi R, Ardalan G, Gheiratmand R, et al. CASPIAN Study Group. Association of physical activity and dietary behaviours in relation to the body mass index in a national sample of Iranian children and adolescents: CASPIAN Study. Bull World Health Organ 2007; 85: 19-26.

[46] Kelishadi R, Gouya MM, Adeli K, et al. CASPIAN Study Group. Factors associated with the metabolic syndrome in a national sample of youths: CASPIAN Study. Nutr Metab Cardiovasc Dis 2008; 18: 461-70.

[47] Roberts CK, Won D, Pruthi S, et al. Effect of a short-term diet and exercise intervention on oxidative stress, inflammation, MMP-9, and monocyte chemotactic activity in men with metabolic syndrome factors. J Appl Physiol 2006; 100: 1657-65.

[48] Kelishadi R, Hashemi M, Mohammadifard N, Asgary S, Khavarian $\mathrm{N}$. Association of changes in oxidative and proinflammatory states with changes in vascular function after a lifestyle modification trial among obese children. Clin Chem 2008; 54: 147-53.

[49] Kelishadi R, Cook SR, Motlagh ME, et al. Metabolically obese normal weight and phenotypically obese metabolically normal youths: the CASPIAN Study. J Am Diet Assoc 2008; 108: 82-90.

[50] Kelishadi R, Cook SR, Amra B, Adibi A. Factors associated with insulin resistance and non-alcoholic fatty liver disease among youths. Atherosclerosis 2009; 204: 538 -43.

[51] Kelishadi R, Hashemipour M, Sarrafzadegan N, et al. Effects of a lifestyle modification trial among phenotypically obese metabolically normal and phenotypically obese metabolically abnormal adolescents in comparison with phenotypically normal metabolically obese adolescents. J Mater Child Nutr 2009 (in press).

[52] Juonala M, Viikari JS, Ronnemaa T, Taittonen L, Marniemi J, Raitakari OT. Childhood C-reactive protein in predicting CRP and carotid intima-media thickness in adulthood. The Cardiovascular Risk in Young Finns Study. Arterioscler Thromb Vasc Biol 2006; 26: $1883-8$ 
[53] Mattsson N, Ronnemaa T, Juonala M, Viikari JS, Raitakari OT. Childhood predictors of the metabolic syndrome in adulthood. The Cardiovascular Risk in Young Finns Study. Ann Med 2008; 40: 542-52.

[54] Kelishadi R, Zadegan NS, Naderi GA, Asgary S, Bashardoust N. Atherosclerosis risk factors in children and adolescents with or without family history of premature coronary artery disease. Med Sci Monit 2002; 8: 425-9.

[55] Kelishadi R, Nadery GA, Asgary S. Oxidized LDL metabolites with high family risk for premature cardiovascular disease. Indian J Pediatr 2002; 69: 755-9.

[56] Magadle R, Merlon H, Weiner P, Mohammedi I, Robert D. Creactive protein levels and arterial abnormalities in the offspring of patients with premature myocardial infarction. Cardiology 2003; 100: 1-6.

[57] Kelishadi R, Sabet B, Khosravi A. Anticardiolipin antibody of adolescents and age of myocardial infarction in parents. Med Sci Monit 2003; 9: 515-8.

[58] Gaeta G, De Michele M, Cuomo S, et al. Arterial abnormalities in the offspring of patients with premature myocardial infarction. $\mathrm{N}$ Engl J Med 2000; 343: 840-6.

[59] Cuomo S, Guarini P, Gaeta G, et al. Increased carotid intima-media thickness in children-adolescents, and young adults with a parental history of premature myocardial infarction. Eur Heart J 2002; 23: 1345-50.

[60] Sabri MR, Kelishadi R. The thickness of the intimal and medial layers of the carotid arteries, and the index of left ventricular mass, in children of patients with premature coronary arterial disease. Cardiol Young 2007; 17: 609-16.

[61] Kelishadi R, Sabri M, Motamedi N, Ramezani MA. Factor analysis of markers of inflammation and oxidation and echocardiographic findings in children with a positive family history of premature coronary heart disease. Pediatr Cardiol 2009; 30: 477-81.

[62] de Ferranti SD, Rifai N. C-reactive protein: a nontraditional serum marker of cardiovascular risk. Cardiovasc Pathol 2007; 16: 1421.

[63] Abramson JL, Hooper WC, Jones DP, et al. Association between novel oxidative stress markers and C-reactive protein among adults without clinical coronary heart disease. Atherosclerosis 2005; 178: 115-21.

[64] Fujii H, Li SH, Szmitko PE, Fedak PW, Verma S. C-reactive protein alters antioxidant defenses and promotes apoptosis in endothelial progenitor cells. Arterioscler Thromb Vasc Biol 2006; 26: 2476-82.

[65] Stoll G, Bendszus M. Inflammation and atherosclerosis: novel insights into plaque formation and destabilization. Stroke 2006; 37 : 1923-32.

[66] Libby P, Ridker PM, Maseri A. Inflammation and atherosclerosis. Circulation 2002; 106: 135-6.
[67] Jacobs M, van Greevenbroek MM, van der Kallen CJ, et al. Lowgrade inflammation can partly explain the association between the metabolic syndrome and either coronary artery disease or severity of peripheral arterial disease: the CODAM study. Eur J Clin Invest 2009 Apr 9. [Epub ahead of print]

[68] Puddu P, Muscari A, Puddu GM, Cravero E, Giannoni C, Zoli M. The complexity of platelet metabolism and its contribution to atherothrombosis. Acta Cardiol 2009; 64: 157-65.

[69] Wagner DD, Burger PC. Platelets in inflammation and thrombosis. Arterioscler Thromb Vasc Biol 2003; 23: 2131-7.

[70] Huo Y, Schober A, Forlow SB, et al. Circulating activated platelets exacerbate atherosclerosis in mice deficient in apolipoprotein $\mathrm{E}$. Nat Med 2003; 9: 61-7.

[71] Weber C, Schober A, Zernecke A. Chemokines key regulators of mononuclear cell recruitment in atherosclerotic vascular disease. Arterioscler Thromb Vasc Biol 2004; 24:1997-2008.

[72] Sprague AH, Khalil RA. Inflammatory cytokines in vascular dysfunction and vascular disease. Biochem Pharmacol 2009 May 3. [Epub ahead of print]

[73] Weber C. Platelets and chemokines in atherosclerosis: partners in crime. Circ Res 2005; 96: 612-6.

[74] Gasparyan AY, Watson T, Lip GY. The role of aspirin in cardiovascular prevention:implications of aspirin resistance. J Am Coll Cardiol 2008; 51: 1829-43.

[75] Wang L, Lu X, Li Y, Li H, Chen S, Gu D. Functional analysis of the $\mathrm{C}$-reactive protein (CRP) gene $-717 \mathrm{~A}>\mathrm{G}$ polymorphism associated with coronary heart disease. BMC Med Genet 2009; 10: 73.

[76] Singh V, Tiwari RL, Dikshit M, Barthwal MK. Models to study atherosclerosis: a mechanistic insight. Curr Vasc Pharmacol 2009; 7: 75-109.

[77] Elliott P, Chambers JC, Zhang W, et al. Genetic Loci associated with C-reactive protein levels and risk of coronary heart disease. JAMA 2009; 302: 37-48.

[78] Chen YC, Chen YD, et al. The HMG-CoA reductase gene and lipid and lipoprotein levels: the Multi-Ethnic study of atherosclerosis. Lipids 2009; 44: 733-43.

[79] Kavey RE, Daniels SR, Lauer RM, Atkins DL, Hayman LL, Taubert K. American Heart Association. American Heart Association guidelines for primary prevention of atherosclerotic cardiovascular disease beginning in childhood. Circulation 2003; 107: 1562-6.

[80] Gidding SS, Lichtenstein AH, Faith MS, et al. Implementing American Heart Association pediatric and adult nutrition guidelines: a scientific statement from the American Heart Association Nutrition Committee of the Council on Nutrition, Physical Activity and Metabolism, Council on Cardiovascular Disease in the Young, Council on Arteriosclerosis, Thrombosis and Vascular Biology, Council on Cardiovascular Nursing, Council on Epidemiology and Prevention, and Council for High Blood Pressure Research. Circulation 2009; 119: 1161-75. 